SLAC-PUB-8756

hep-ph/0101228

\title{
Radiative corrections to the Casimir force and effective field theories
}

\author{
Kirill Melnikov* \\ Stanford Linear Accelerator Center \\ Stanford University, Stanford, CA 94309
}

\begin{abstract}
Radiative corrections to the Casimir force between two parallel plates are considered in both scalar field theory of one massless and one massive field and in QED. Full calculations are contrasted with calculations based on employing "boundary-free" effective field theories. The difference between two previous results on QED radiative corrections to the Casimir force between two parallel plates is clarified and the low-energy effective field theory for the Casimir effect in QED is constructed.
\end{abstract}

*e-mail: melnikov@slac.stanford.edu 


\section{INTRODUCTION}

Attraction of two infinitely conducting plates caused by vacuum fluctuations was predicted by H. Casimir in 1948 [㑑]. Experimental attempts to verify this prediction have started almost immediately, however, it took almost half a century to reach conclusive and accurate outcome. To date the Casimir effect is verified on a few per cent level [2, [3].

Recent theoretical work on the Casimir effect has been focused around several topics [4] such as calculation of the Casimir force for complicated geometries, material dependence and the study of radiative corrections to the Casimir force in QED. The latter studies resulted in an interesting controversy. The one-loop QED radiative corrections to the Casimir force in case of two infinitely conducting parallel plates have been computed in [5] and have been found to be $\mathcal{O}(\alpha /(m L))$ relative to the leading order result ( $m$ is the electron mass, $L$ is the distance between the two plates and $\alpha$ is the fine structure constant). Later on, the problem was reconsidered in [6]. The authors of Ref. [6] argued that, since the Casimir force is the long-distance effect with a typical photon momentum being of the order $\sim 1 / L$, it is natural to integrate out the momentum scales comparable to the electron mass and to use the effective field theory of QED (in essence, the Euler-Heisenberg Lagrangian) to compute radiative corrections to the Casimir force. That calculation resulted in the relative correction $\mathcal{O}\left(\alpha^{2} /(m L)^{4}\right)$ that is higher order in $\alpha$, analytic in the mass of the electron squared and much more strongly suppressed by the distance between the two plates than the results of [5].

To the best of my knowledge, the issue has never been clarified (see e.g. the recent discussion in [7]) and it is the aim of this paper to present a clarification. To avoid unnecessary complications, I begin by considering the theory of two interacting scalar fields, one massive and one massless, and compute the radiative corrections to the Casimir force in case of two plates separated by the distance $L$. Then I generalize these results on the Casimir effect in QED円.

I will start with the detailed description of the full calculation of the one-loop corrections to the Casimir force. Similar to [5, 6], the massless fields satisfy certain boundary conditions on the plates but no boundary conditions are imposed on the massive fields. Due to different quantization conditions on massive and massless fields, the calculation of radiative corrections to the Casimir force is similar to the calculation of scattering processes in an external background field (whose exact form is determined by the boundary conditions on the massless field) that facilitates virtual transitions from massless to massive fields. It follows from this that boundary-free effective field theories do not completely account for the low-energy limit of the problem, since they are derived in the absence of a non-trivial external background. This observation invalidates the results of [6] as being derived from the wrong effective field theory. Finally, I construct the effective field theory appropriate for the calculation of the Casimir force between two infinitely conducting parallel plates.

\footnotetext{
${ }^{1}$ Note that the radiative corrections to the Casimir force are of no phenomenological significance. The interest in the problem is primarily related to such theoretical issue as the quantum field theory with non-trivial boundary conditions.
} 


\section{THE CASIMIR FORCE}

Consider the theory of two scalar fields $\phi$ and $\chi$ whose dynamics is governed by the Lagrangian:

$$
\mathcal{L}=\frac{1}{2}\left(\partial_{\mu} \phi\right)^{2}+\frac{1}{2}\left(\partial_{\mu} \chi\right)^{2}-\frac{1}{2} M^{2} \chi^{2}-\frac{g}{2} \phi \chi^{2}
$$

External conditions are set by two parallel plates located at the points $z=0$ and $z=L$. The Dirichlet boundary conditions are imposed on the field $\phi$, i.e. $\left.\phi\right|_{z=0}=\left.\phi\right|_{z=L}=0$. We do not impose any boundary conditions on the massive field $\chi$. The Green's function of the massless field $\phi$ is given by:

$$
G\left(t, \boldsymbol{r}_{\perp}, z, z^{\prime}\right)=\frac{2 i}{L} \int \frac{d \omega}{2 \pi} \frac{d^{d} k_{\perp}}{(2 \pi)^{d}} e^{-i \omega t+i \boldsymbol{k}_{\perp} \boldsymbol{r}_{\perp}} \sum_{n=1} \frac{\sin (\kappa n z) \sin \left(\kappa n z^{\prime}\right)}{\omega^{2}-\boldsymbol{k}_{\perp}^{2}-(\kappa n)^{2}+i \delta}
$$

with $\kappa=\pi / L$ and $\boldsymbol{k}_{\perp}$ and $\boldsymbol{r}_{\perp}$ being momentum and position vectors that are parallel to the plates. I intend to use dimensional regularization for the calculations in what follows; for this reason the transverse space is considered to be $d$-dimensional, with $d=2-2 \epsilon$ where $\epsilon$ is the regularization parameter.

The quantity of interest is the vacuum energy per unit transverse volume between the two plates. It can be obtained from $T_{00}$ component of the stress-energy tensor (which is the Hamiltonian density of the system):

$$
T_{00}=\frac{1}{2}\left(\partial_{0} \phi\right)^{2}+\frac{1}{2}(\boldsymbol{\partial} \phi)^{2}+\frac{1}{2}\left(\partial_{0} \chi\right)^{2}+\frac{1}{2}(\boldsymbol{\partial} \chi)^{2}+\frac{1}{2} M^{2} \chi^{2}+\frac{g}{2} \chi^{2} \phi
$$

We then compute:

$$
E=\int_{0}^{L} \mathrm{~d} z\left\langle 0\left|T_{00}(z)\right| 0\right\rangle
$$

Consider first the leading order contribution. In momentum space it reads:

$$
E=\sum_{n=1} \int \frac{d \omega}{2 \pi} \frac{d^{d} k_{\perp}}{(2 \pi)^{d}} \frac{i}{\omega^{2}-\boldsymbol{k}_{\perp}^{2}-(\kappa n)^{2}+i \delta} \frac{\omega^{2}+\boldsymbol{k}_{\perp}^{2}+(\kappa n)^{2}}{2} .
$$

To compute $E$ in the above equation we first integrate over $\omega$ by taking the residues (it is important to keep in mind that scaleless integrals in dimensional regularization are set to zero). The result is:

$$
E=\frac{1}{2} \sum_{n=1} \int \frac{d^{d} k_{\perp}}{(2 \pi)^{d}} \sqrt{\boldsymbol{k}_{\perp}^{2}+(\kappa n)^{2}} .
$$

To proceed further, we rescale $\left|\boldsymbol{k}_{\perp}\right| \rightarrow\left|\boldsymbol{k}_{\perp}\right| \kappa n$; after that the integration over $k_{\perp}$ and the summation over $n$ factorize. Finally, we obtain the well-known result:

$$
E=\frac{1}{2}\left(\frac{\pi}{L}\right)^{d+1} \zeta(-3+2 \epsilon) \frac{\Gamma(-3 / 2+\epsilon)}{(4 \pi)^{d / 2} \Gamma(-1 / 2)}=-\frac{\pi^{2}}{1440 L^{3}} .
$$


Note that, in principle, we should subtract from the above result the vacuum energy computed in the boundary-free field theory. However, it is easy to see that for the massless field such a contribution vanishes (since it is given by a scaleless integral). For this reason Eq.(1) gives the complete result for the Casimir vacuum energy. In general, we will understand the subtraction of boundary-free contributions as the result of adding position independent counterterms to the Hamiltonian densityt. These counterterms are computed in boundary-free field theory and they are constructed in such a way that the vacuum energy density in the boundary-free case vanishes.

The Casimir force between the two plates is minus derivative of the vacuum energy with respect to the distance between the plates. One obtains:

$$
f_{0}=-\frac{\partial}{\partial L} E=-\frac{\pi^{2}}{480 L^{4}}
$$

\section{RADIATIVE CORRECTIONS}

We now turn to the consideration of the radiative corrections to the Casimir force. There are three diagrams that contribute; they are shown in Fig.1. Note that because of the form of the Lagrangian Eq.(伊), the self-interaction of the field $\phi$ is of higher order in the coupling constant and for this reason is not considered here.

We begin with the diagram Fig.1a, that describes the vacuum polarization of the massless field $\phi$ caused by the massive field $\chi$. Since massless and massive fields are quantized differently, there is a peculiarity in writing down expressions for Feynman diagrams in that one does not expect the momentum conservation to hold for all interaction vertices. On the other hand, it is clear how the perturbative expansion in position space is constructed and we can derive the momentum representation if we start from there. The expression for the vacuum energy in position space is:

$$
\delta E^{(1 a)} \sim g^{2} \int_{0}^{L} \mathrm{~d} z \mathrm{~d} z_{1} \mathrm{~d} z_{2} G\left(z, z_{1}\right) P\left(z_{1}-z_{2}\right)^{2} G\left(z_{2}, z\right),
$$

where $P(z)$ is the propagator of the field $\chi$. The important point is that all the integrations range from 0 to $L$ since this is the only region where the Green's function $G$ has the nonvanishing support.

Because of the boundary conditions, the eigenfunctions of the $\chi$ field are not orthogonal to the eigenfunctions of the $\phi$ field; as a consequence we get momentum non-conservation in the $\phi \chi^{2}$ interaction vertex. Switching to the momentum space in Eq.(9)), one finds the following representation for $\delta E^{(1 a)}$ :

\footnotetext{
${ }^{2}$ Because of the normal ordering prescription, these counterterms are usually not included into boundary-free field theory calculations.
} 


$$
\delta E^{(1 a)}=-\frac{i}{2} \sum_{n=1} \int \frac{d \omega}{2 \pi} \int \frac{\mathrm{d} \Delta}{(2 \pi)} \frac{d^{d} k_{\perp}}{(2 \pi)^{d}} \frac{\left(\omega^{2}+\boldsymbol{k}_{n}^{2}\right)}{2} \frac{1}{\left(\omega^{2}-\boldsymbol{k}_{n}^{2}+i \delta\right)^{2}} \mathcal{F}_{D}(\kappa n, \Delta) \Pi_{M}\left(\omega, \boldsymbol{k}_{\perp}, \Delta\right),
$$

where

$$
\mathcal{F}_{D}(\kappa n, \Delta)=\frac{4(\kappa n)^{2}}{L} \frac{(1-\cos (\kappa n L) \cos (\Delta L))}{\left(\Delta^{2}-(\kappa n)^{2}+i \delta\right)^{2}}, \quad \boldsymbol{k}_{n}=\left(\boldsymbol{k}_{\perp}, \kappa n\right)
$$

and $i \Pi_{M}$ is the vacuum polarization function?. It is apparent from Eq.(10) that there is a mismatch in the momentum that flows along the $\phi$ line and the momentum that goes into the vacuum polarization. The mismatch is described by the function $\mathcal{F}_{D}(\kappa n, \Delta)$; this function is peaked at $|\Delta|=\kappa n$, but it differs from zero for all other values of $\Delta$ and $\kappa n$. Note also that, for fixed boundary conditions, the function $\mathcal{F}_{D}$ is universal and will appear in all the other diagrams we consider.

To proceed further, it is convenient to use the dispersion representation for the vacuum polarization function:

$$
\Pi_{M}(q)=\frac{1}{\pi} \int_{4 M^{2}}^{\infty} \frac{\mathrm{d} s \operatorname{Im} \Pi_{M}(s)}{s-q^{2}-i \delta}
$$

where $q^{2}=\omega^{2}-\boldsymbol{k}_{\perp}^{2}-\Delta^{2}$ and the imaginary part is defined as:

$$
\operatorname{Im} \Pi_{M}(s)=\frac{g^{2}}{(2 \pi)^{D}} \frac{\pi^{7 / 2-\epsilon}}{2^{1-2 \epsilon} \Gamma(3 / 2-\epsilon)} s^{-\epsilon}\left(1-\frac{4 M^{2}}{s}\right)^{1 / 2-\epsilon} .
$$

We now see that the integration over $\Delta$ in Eq.(10) can be performed by using the residue theorem. There are two different contributions: one from $\Delta= \pm \kappa n$ and another from $\Delta= \pm \sqrt{s-\omega^{2}+\boldsymbol{k}_{\perp}^{2}}$. Let us consider these two contributions separately. In view of the fact that the integral is symmetric with respect to the transformation $\Delta \rightarrow-\Delta$, we can substitute $\cos (\Delta L) \rightarrow e^{i \Delta L}$ in the function $\mathcal{F}_{D}$ and close the integration contour in the upper half plane.

Consider first the contribution that comes from the second order residue at $\Delta=-\kappa n+i \delta$. Since $\kappa n L=\pi n$, the only possibility to get a non-zero contribution is to differentiate $\left(1-\cos (\kappa n L) e^{i \Delta L}\right)$ in the function $\mathcal{F}_{D}$ with respect to $\Delta$. We obtain:

$$
\delta E_{1}^{(1 a)}=-\frac{i}{2} \sum_{n=1} \int \frac{d \omega}{2 \pi} \frac{d^{d} k_{\perp}}{(2 \pi)^{d}} \frac{\left(\omega^{2}+\boldsymbol{k}_{n}^{2}\right)}{2} \frac{1}{\left(\omega^{2}-\boldsymbol{k}_{n}^{2}+i \delta\right)^{2}} \Pi_{M}\left(\omega, k_{n}\right) .
$$

Note that this is exactly the contribution one would write down if momentum conservation in $\phi \chi^{2}$ vertex is used blindly.

\footnotetext{
${ }^{3}$ The $i \delta$ prescription introduced in the function $\mathcal{F}_{D}$ is for the integration convenience; it can be changed in an arbitrary way since the function is finite at $\Delta= \pm \kappa n$ because $\cos (\kappa L n)=(-1)^{n}$.
} 
To ensure that the field $\phi$ stays massless one has to add a mass counterterm to the above expression. Note, that since the subtraction is done at $q^{2}=0, \delta E_{1}^{(1 a)}$ is the right place to add the mass counterterm contribution. It is also convenient to perform the Wick rotation $\omega \rightarrow i \omega$ at this step. We then obtain:

$$
\delta E_{1}^{(1 a)}=\frac{1}{2} \sum_{n=1} \int \frac{d \omega}{2 \pi} \frac{d^{d} k_{\perp}}{(2 \pi)^{d}} \frac{\left(\omega^{2}-\boldsymbol{k}_{n}^{2}\right)}{2 k_{n}^{2}} \frac{1}{\pi} \int_{4 M^{2}}^{\infty} \frac{\mathrm{d} s \operatorname{Im} \Pi_{M}(s)}{s\left(s+k_{n}^{2}\right)}
$$

where $k_{n}=\left(\omega, \boldsymbol{k}_{n}\right)$. The simplest way to proceed further is to do the partial fractioning to obtain:

$$
\frac{\left(\omega^{2}-\boldsymbol{k}_{n}^{2}\right)}{2 k_{n}^{2}} \frac{1}{\left(s+k_{n}^{2}\right)}=\frac{\omega^{2}}{s} \frac{1}{k_{n}^{2}}-\frac{1}{2}\left(1+\frac{2 \omega^{2}}{s}\right) \frac{1}{k_{n}^{2}+s} .
$$

We now consider the two terms separately. The first one is:

$$
\delta E_{11}^{(1 a)}=\frac{1}{2} \sum_{n=1} \int \frac{d \omega}{2 \pi} \frac{d^{d} k_{\perp}}{(2 \pi)^{d}} \frac{\omega^{2}}{k_{n}^{2}} \frac{1}{\pi} \int_{4 M^{2}}^{\infty} \frac{\mathrm{d} s \operatorname{Im} \Pi_{M}(s)}{s^{2}} .
$$

Taking into account that scaleless integrals in dimensional regularization are set to zero, one immediately recognizes that this contribution is related to the wave function renormalization of the massless field and is therefore not relevant.

To deal with the second term in the r.h.s. of Eq.(16), we first perform the sum over $n$ and neglect the terms that are exponentially suppressed $\leq \mathcal{O}\left(e^{-2 L M}\right)$ (cf. the complete formula in Appendix):

$$
\sum_{n=1}^{\infty} \frac{1}{\omega^{2}+\boldsymbol{k}_{\perp}^{2}+(\kappa n)^{2}+s}=\frac{L}{2 \sqrt{\omega^{2}+\boldsymbol{k}_{\perp}^{2}+s}}-\frac{1}{2\left(\omega^{2}+\boldsymbol{k}_{\perp}^{2}+s\right)}+\mathcal{O}\left(e^{-2 L M}\right) .
$$

We see that the middle term in the right hand side of Eq.(18) is $L$-independent and hence does not contribute to the Casimir force. On the other hand, the first term in the r.h.s. of Eq.(18) is nothing but the integral:

$$
L \int \frac{\mathrm{d} k_{z}}{(2 \pi)} \frac{1}{\omega^{2}+\boldsymbol{k}_{\perp}^{2}+k_{z}^{2}+s}=\frac{L}{2 \sqrt{\omega^{2}+\boldsymbol{k}_{\perp}^{2}+s}}
$$

and hence its contribution to the vacuum energy can be written as:

$$
\delta E_{12}^{(1 a)}=-\frac{L}{4} \int \frac{d \omega}{2 \pi} \frac{d^{d+1} k}{(2 \pi)^{d+1}} \frac{1}{\pi} \int_{4 M^{2}}^{\infty} \frac{\mathrm{d} s \operatorname{Im} \Pi_{M}(s)}{s\left(\omega^{2}+\boldsymbol{k}^{2}+s\right)}\left(1+\frac{2 \omega^{2}}{s}\right) .
$$

A nice feature of this result is that it comes out being proportional to the distance between the two plates and so we can check that it will be canceled by the boundary-free field theory counterterm for the vacuum energy density. The boundary-free vacuum energy is given by the expression: 


$$
\delta E_{\text {free }}^{(1 a)}=-\frac{i L}{2} \int \frac{d \omega}{2 \pi} \frac{d^{d+1} k}{(2 \pi)^{d+1}} \frac{\left(\omega^{2}+\boldsymbol{k}^{2}\right)}{2} \frac{1}{\left(\omega^{2}-\boldsymbol{k}^{2}+i \delta\right)^{2}} \Pi_{M}(\omega, \boldsymbol{k}) .
$$

We again use (subtracted) dispersion relation for the vacuum polarization and proceed along the lines described above. We then find the result for $\delta E_{\text {free }}^{(1 a)}$ that exactly matches the result for $\delta E_{12}^{(1 a)}$ in Eq.(20). We therefore conclude that, neglecting exponentially suppressed terms, the "momentum conserving" contribution to the Casimir energy $\delta E_{1}^{(1 a)}$ vanishes upon the subtraction of the boundary-free vacuum energy and appropriate renormalization of the wave function of the massless field.

Hence, we have to concentrate on the second contribution to $\delta E^{(1 a)}$ that comes from the "momentum non-conserving" part of the $\Delta$ integral in Eq.(10). It is convenient to perform the Wick rotation $\omega \rightarrow i \omega$ and then integrate over $\Delta$ picking up the pole at $\Delta=$ $i \sqrt{w^{2}+\boldsymbol{k}_{\perp}^{2}+s}$. We again neglect the exponentially suppressed term $\leq \mathcal{O}\left(e^{-2 M L}\right)$ coming from $\cos (\Delta L)$ and obtain:

$$
\delta E_{2}^{(1 a)}=-\frac{1}{L} \sum_{n=1} \int \frac{d \omega}{2 \pi} \frac{d^{d} k_{\perp}}{(2 \pi)^{d}} \frac{\left(\omega^{2}-\boldsymbol{k}_{n}^{2}\right)}{2\left(k_{n}^{2}\right)^{2}} \frac{1}{\pi} \int_{4 M^{2}}^{\infty} \frac{\mathrm{d} s \operatorname{Im} \Pi_{M}(s)}{\sqrt{s+w^{2}+\boldsymbol{k}_{\perp}^{2}}} \frac{(\kappa n)^{2}}{\left(s+k_{n}^{2}\right)^{2}} .
$$

To compute this integral, we combine the denominators using Feynman parameters and carry out the integration over $\omega$. After appropriate rescaling, we integrate over $k_{\perp}$ and introduce the Mellin transform to perform the sum over $n$. The final result then reads (we do not display the $L$-independent contribution):

$$
\delta E_{2}^{(1 a)}=\frac{1}{24 \pi^{2} L} \int_{4 M^{2}}^{\infty} \frac{\mathrm{d} s \operatorname{Im}_{M}(s)}{s} \sum_{n=1}^{\infty}(2 n+3) \zeta(-2 n-3) \frac{\Gamma(n-1 / 2)}{\sqrt{\pi} \Gamma(n)}\left(\frac{\kappa}{\sqrt{s}}\right)^{2 n+3} .
$$

We explicitly see that all these contributions are finite and depend in a non-analytic way on the mass of the heavy particle squared. The first few terms read:

$$
\delta E_{2}^{(1 a)}=-\frac{5}{12386304} \frac{\pi^{4} \alpha}{M^{3} L^{6}}+\frac{7}{150994944} \frac{\pi^{6} \alpha}{M^{5} L^{8}}
$$

where dimensionless coupling constant $\alpha=g^{2} /\left(4 \pi M^{2}\right)$ is introduced.

The series in Eq.(23) are asymptotic; the generating function can be obtained using the formulas from Appendix:

$$
\delta E^{(1 a)}=\frac{-1}{24 \pi^{2} L} \int_{4 M^{2}}^{\infty} \frac{\mathrm{d} s \operatorname{Im} \Pi_{M}(s)}{s} \frac{1}{T(s) \pi} \int_{0}^{\infty} \frac{\mathrm{d} u}{e^{u / T(s)}-1} \frac{u^{5}\left(5+4 u^{2}\right)}{\left(1+u^{2}\right)^{3 / 2}}
$$

where $T(s)=\kappa /(2 \pi \sqrt{s})$. The above equation completes our discussion of the graph Fig.1a.

Let us consider the graph shown in Fig.1b. In the momentum representation its contribution to the vacuum energy reads:

$$
\delta E^{(1 b)}=-i \sum_{n=1} \int \frac{d \omega}{2 \pi} \int \frac{\mathrm{d} \Delta}{(2 \pi)} \frac{d^{d} k_{\perp}}{(2 \pi)^{d}} \frac{1}{\left(\omega^{2}-\boldsymbol{k}_{n}^{2}+i \delta\right)} \mathcal{F}_{D}(\kappa n, \Delta) V\left(\omega, k_{\perp}, \Delta\right),
$$


where $\mathcal{F}_{D}$ is defined in Eq.(11), the function $i V$ is given by

$$
i V(q)=g^{2} \int \frac{\mathrm{d}^{D} l}{(2 \pi)^{D}} \frac{\left(2(Q l)^{2}-l^{2}+M^{2}\right) / 2}{\left[l^{2}-M^{2}+i \delta\right]^{2}\left[(l+q)^{2}-M^{2}+i \delta\right]},
$$

and $Q$ denotes the time-like vector $Q=(1,0,0,0)$.

We now give an argument that no non-trivial momentum conserving contribution can appear from this graph. The argument goes as follows. Let us perform the $\Delta$ integration by picking up the momentum conserving pole in $\Delta$. We get:

$$
\delta E^{(1 b)}=-i \sum_{n=1} \int \frac{d \omega}{2 \pi} \int \frac{\mathrm{d} \Delta}{(2 \pi)} \frac{d^{d} k_{\perp}}{(2 \pi)^{d}} \frac{1}{\left(\omega^{2}-\boldsymbol{k}_{n}^{2}+i \delta\right)} V\left(k_{n}\right) .
$$

Let us now use the fact that the function $V(k)$ obeys dispersion relations with respect to momentum transfer $k$. The dispersion relations are written in the subtracted form, to ensure that there is no correction to the interaction of the on-shell massless particle with the stress-energy tensor. We then have:

$$
V\left(k_{n}\right)=k_{n}^{2}\left[\frac{1}{\pi} \int_{4 M^{2}}^{\infty} \frac{\mathrm{d} s \operatorname{Im} V_{1}(s)}{s\left(s-k_{n}^{2}-i \delta\right)}+\left(Q k_{n}\right)^{2} \frac{1}{\pi} \int_{4 M^{2}}^{\infty} \frac{\mathrm{d} s \operatorname{Im} V_{2}(s)}{s^{2}\left(s-k_{n}^{2}-i \delta\right)}\right]
$$

where the imaginary parts are given by:

$$
\begin{aligned}
& \operatorname{Im} V_{1}(s)=\frac{-g^{2}}{(2 \pi)^{D}} \frac{\pi^{7 / 2-\epsilon}}{2^{3-2 \epsilon} \Gamma(3 / 2-\epsilon)} s^{-\epsilon}\left(1-\frac{4 M^{2}}{s}\right)^{1 / 2-\epsilon} \\
& \operatorname{Im} V_{2}(s)=\frac{g^{2}}{(2 \pi)^{D}} \frac{\pi^{7 / 2-\epsilon}}{2^{2-2 \epsilon} \Gamma(3 / 2-\epsilon)}\left(\epsilon-\frac{2 M^{2}}{s}\right) s^{-\epsilon}\left(1-\frac{4 M^{2}}{s}\right)^{-1 / 2-\epsilon}
\end{aligned}
$$

If we substitute this expression to Eq.(28), we cancel the massless particle propagator; the only other "propagator" left is $1 /\left(s-k_{n}^{2}\right)$. Similar to the case of the vacuum polarization graph discussed above, the sum over $n$ can be performed; disregarding the exponentially suppressed contribution and also the contribution which is $L$ independent, this sum appears equivalent to the integral over $k_{z}$. It is then easy to see that this contribution exactly matches the boundary-free vacuum energy and gets subtracted completely.

Therefore, the only non-trivial contribution to the vacuum energy from Fig.1b is again related to the momentum configuration where $\Delta$ is of the order of the mass of the heavy particle. To compute this contribution we proceed in a way similar to what has been done for the vacuum polarization graph Fig.1a and obtain:

$$
\begin{aligned}
\delta E^{(1 b)} & =\frac{1}{2 \pi^{2} L} \int_{4 M^{2}}^{\infty} \frac{\mathrm{d} s \operatorname{Im} V_{1}(s)}{s} \sum_{n=1}^{\infty} \zeta(-2 n-1) \frac{\Gamma(n-1 / 2)}{\sqrt{\pi} \Gamma(n)}\left(\frac{\kappa}{\sqrt{s}}\right)^{2 n+1} \\
& +\frac{1}{3 \pi^{2} L} \int_{4 M^{2}}^{\infty} \frac{\mathrm{d} s \operatorname{Im} V_{2}(s)}{s} \sum_{n=1}^{\infty} \zeta(-2 n-1) \frac{\Gamma(n-1 / 2)}{\sqrt{\pi} \Gamma(n)} \frac{(n-1)}{(2 n-3)}\left(\frac{\kappa}{\sqrt{s}}\right)^{2 n+1} .
\end{aligned}
$$


Again, the series are asymptotic; the generating function for these series can be easily established in a manner similar to what has lead to Eq.(25). The first few terms of $\delta E^{(16)}$ read:

$$
\delta E^{(1 b)}=-\frac{1}{245760} \frac{\pi^{2} \alpha}{L^{4} M}+\frac{13}{24772608} \frac{\pi^{4} \alpha}{L^{6} M^{3}} .
$$

The final contribution one has to consider comes from the diagram shown in Fig.1c and reads:

$$
\delta E^{(1 c)}=-\frac{i}{2} \sum_{n=1} \int \frac{d \omega}{2 \pi} \int \frac{\mathrm{d} \Delta}{(2 \pi)} \frac{d^{d} k_{\perp}}{(2 \pi)^{d}} \frac{1}{\left(\omega^{2}-\boldsymbol{k}_{n}^{2}+i \delta\right)} \mathcal{F}_{D}(\kappa n, \Delta) \Pi_{M}\left(\omega, k_{\perp}, \Delta\right) .
$$

This contribution is easily analyzed following the discussion of the vacuum polarization graph Fig.1a at the beginning of this Section. Again, the only $L$-dependent contribution that does not get canceled against the corresponding boundary-free field theory counterterm is related to large values of $\Delta$. The full result is:

$$
\delta E^{(1 c)}=\frac{1}{4 \pi^{2} L} \int_{4 M^{2}}^{\infty} \frac{\mathrm{d} s \operatorname{Im} \Pi_{M}(s)}{s} \sum_{n=1}^{\infty} \zeta(-2 n-1) \frac{\Gamma(n-1 / 2)}{\sqrt{\pi} \Gamma(n)}\left(\frac{\kappa}{\sqrt{s}}\right)^{2 n+1},
$$

and the first few terms read:

$$
\delta E^{(1 c)}=\frac{1}{122880} \frac{\pi^{2} \alpha}{L^{4} M}-\frac{1}{4128768} \frac{\pi^{4} \alpha}{L^{6} M^{3}} .
$$

The sum of the above results Eqs. $(24,33,36)$ gives the correction to the vacuum energy:

$$
\delta E^{(1)}=\frac{1}{245760} \frac{\pi^{2} \alpha}{L^{4} M}+\mathcal{O}\left(\alpha L^{-6} M^{-3}\right) .
$$

The relative correction to the Casimir force then becomes:

$$
\frac{\delta f}{f_{0}}=-\frac{\alpha}{128 L M},
$$

where $f_{0}$ is the leading order result Eq.(8). Let me emphasize that this contribution is non-analytic in the mass of the heavy particle squared and in this respect is similar to the result reported in [国陵.

\section{EFFECTIVE FIELD THEORY DESCRIPTION}

My main motivation for considering this problem was the desire to clarify the apparent discrepancy between the full and the effective field theory calculations of the QED radiative

\footnotetext{
${ }^{4}$ To avoid confusion, Eq.(38) is derived in the context of the scalar field theory, whereas the calculation of Ref. [5] is done for QED.
} 
corrections to the Casimir energy in case of two infinitely conducting parallel plates. Having performed an explicit full calculation in the previous Section, we can establish what went wrong with the effective field theory arguments as applied in Ref. [6] and the way to modify them to make the effective field theory approach work.

Our key (and rather simple) observation is the fact that the momentum in the heavylight vertex is not conserved, as follows from different quantization conditions on massless and massive fields. In this situation one can think about heavy-light transitions as being induced by external potential. To compute the total energy, one has to integrate over the momentum transfer $\Delta$ from the external potential to the quantum fields. This momentum transfer can be both small $\Delta \sim L^{-1}$ and large $\Delta \sim M$.

When the momentum transfer is small $\Delta \sim L^{-1}$, one can use boundary-free effective field theory, produced by integrating out heavy particles, in the calculation of the Casimir force. From the diagrammatic viewpoint the "hard" (with $k \sim M$ ) subgraph in this case is the loop made up of only massive lines (see Fig.2 ) and is clearly the same as in case of boundary-free field theory. This momentum configuration does produce some effective Lagrangian; however, as we have seen in the previous Section it has no effect on the Casimir force if the usual renormalization program is adopted.

Let me note in passing that, in principle, there is another contribution coming from the momentum conserving piece of the interaction. It corresponds to the situation when the momentum of the massless particle becomes large ( in other words, it corresponds to large values of $n$ in the sum). As I have shown in the previous Section, if one neglects the exponentially suppressed terms and the terms that are $L$-independent, the contribution coming from this momentum region matches exactly the boundary free contribution to the vacuum energy. The trick there was to use the dispersion relations with the cut starting from $4 M^{2}$ and it is not completely clear to me how this particular contribution can be argued away in a more general case.

The configuration with large momentum transfer $\Delta \sim M$ does not have an analogy in the boundary-free case; it is peculiar to the existence of the non-trivial potential. However, apart from the very existence of this contribution, there is nothing special about it and one can treat it along conventional lines of the effective field theories; the change is in the subgraphs that determine effective field theory operators with their Wilson coefficients.

Provided that dimensional regularization is used for the calculations, it is easy to give a general prescription for computing this contribution in a simple way. Following the general pattern of asymptotic expansions of Feynman diagrams, one has to identify small and large quantities for a given momentum configuration and then Taylor expand in all the small quantities. For the case $\Delta \sim M$, the small quantities are the momentum components of the massless field and so one has to Taylor expand in these variables and integrate over the large components of the loop momenta. One then explicitly generates a set of local operators with corresponding Wilson coefficients. The vacuum polarization (Fig.1a) induces the following contribution to the effective Lagrangian:

$$
\delta \mathcal{L}=-\frac{\alpha}{512 L M} \phi\left[\partial_{z}^{2}\right] \phi+\frac{\alpha}{8192 M^{3} L} \phi\left[\partial_{z}^{4}+5 \partial^{2} \partial_{z}^{2}\right] \phi
$$

The other two graphs (Fig1.b,c) represent the renormalization of the $T_{00}$ component of the stress-energy tensor. The corresponding shift in $T_{00}$ can be either computed directly or 
obtained from $\delta \mathcal{L}$ in the standard way.

Let me repeat that the complete low energy effective field theory for the field $\phi$ also includes terms in addition to those shown in $\delta \mathcal{L}$. These terms are $L$-independent, analytic in the mass of the heavy particle squared and can be derived by working with the boundaryfree field theory. As I have shown in the previous Section, those terms do not produce any non-trivial $L$-dependence and are therefore not relevant for the Casimir force.

Finally, let me show that the effective Lagrangian $\delta \mathcal{L}$ does indeed produce the required correction to the Casimir force. To that end, we consider two sources of the correction: the first one is the correction to the Green's function of the $\phi$ field induced by the effective Lagrangian $\delta \mathcal{L}$ Eq.(39); the second one is the correction to the stress-energy tensor.

Consider the change in the vacuum energy caused by the correction to the Green's function of the field $\phi$. We can write it in the following way:

$$
\delta E_{\mathrm{eff}}^{(1 a)}=-\sum_{n=1} \int \frac{d \omega}{2 \pi} \frac{d^{d} k_{\perp}}{(2 \pi)^{d}} \frac{\omega^{2}+\boldsymbol{k}_{n}^{2}}{2\left(k_{n}^{2}\right)^{2}}\left(\frac{i \alpha}{256 L M}(\kappa n)^{2}+\frac{i \alpha}{4096 M^{3} L}\left[(\kappa n)^{4}+5(\kappa n)^{2} k_{n}^{2}\right]\right) .
$$

Further calculation is easy if one performs the Wick rotation and integrates over $\omega$ and $\boldsymbol{k}_{\perp}$. The leading $\mathcal{O}\left(M^{-1}\right)$ contribution in the above equation then vanishes and the result reads:

$$
E_{\text {eff }}^{(1 a)}=-\frac{5}{12386304} \frac{\pi^{4} \alpha}{M^{3} L^{6}},
$$

in agreement with the first term in Eq.(24).

The second contribution occurs because of the change in the stress-energy tensor (it corresponds to graphs Fig.1b,c) which can be easily derived from $\delta \mathcal{L}$ :

$$
\delta T_{00}=\frac{\alpha}{512 L M} \phi\left[\partial_{z}^{2}\right] \phi+\frac{\alpha}{8192 M^{3} L} \phi\left[-\partial_{z}^{4}+5\left(\partial_{0}^{2}+\partial^{2}\right) \partial_{z}^{2}\right] \phi .
$$

Such a change in $T_{00}$ produces the following shift in the vacuum energy:

$$
\delta E_{\mathrm{eff}}^{(1 b)}=\sum_{n=1} \int \frac{d \omega}{2 \pi} \frac{d^{d} k_{\perp}}{(2 \pi)^{d}} \frac{i}{k_{n}^{2}}\left(-\frac{\alpha}{512 L M}(\kappa n)^{2}+\frac{\alpha}{8192 M^{3} L}\left[5\left(\omega^{2}+\boldsymbol{k}_{n}^{2}\right)(\kappa n)^{2}-(\kappa n)^{4}\right]\right) .
$$

The remaining calculation is a simple matter and one obtains:

$$
\delta E_{\mathrm{eff}}^{(1 b)}=\frac{1}{245760} \frac{\pi^{2} \alpha}{L^{4} M}+\frac{1}{3538944} \frac{\pi^{2} \alpha}{M^{3} L^{6}} .
$$

Again, we find an agreement between this result and the sum of Eq.(33) and Eq.(36). We therefore see that it is possible to derive an effective field theory which produces corrections to the Casimir force that are in agreement with the full calculation.

\section{RADIATIVE CORRECTIONS IN QED}

With the understanding gained by considering the scalar field theory example above, it is now a relatively simple matter to compute the radiative corrections to the Casimir force 
in QED. The only difference with the scalar field theory is that the quantization procedure is more tedious. For this reason I am going to spell it out in some detail.

We consider two infinitely conducting plates and impose standard boundary conditions on electric and magnetic fields:

$$
\boldsymbol{E}_{\perp}=0, \quad \boldsymbol{B}_{z}=0 .
$$

The electron field is allowed to propagate freely. This set of boundary conditions is the same as in Refs. [5, 6].

I quantize the theory in the Coulomb gauge. For transverse degrees of freedom, the required formalism has been developed in [8] where explicit expressions for the vector potentials satisfying boundary conditions Eq.(45) have been given. Using these results, we obtain the following propagator for the transverse photon field:

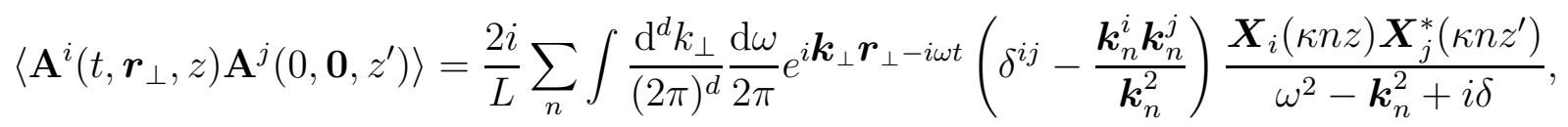

where the vector $\boldsymbol{X}$ is given by:

$$
\boldsymbol{X}=[i \sin (\kappa n z), i \sin (\kappa n z), \cos (\kappa n z)]
$$

Note that the normalization $2 / L$ shown in Eq.(46) only applies to modes with $n \geq 1$. However, for the $z$-component of the vector potential, there is a zero mode with $n=0$; its normalization factor is two times smaller. The zero mode will not be of any concern for us in what follows and for this reason I use a (slightly misleading) representation for the photon propagator as in Eq.(46).

Let me now derive the leading order result for the Casimir force in QED. The photon part of the $T_{00}$ component of the stress-energy tensor is:

$$
T_{00}=\frac{1}{2}\left[\left(\partial_{0} \boldsymbol{A}\right)^{2}+\sum_{i=1}^{3}\left(\partial_{i} \boldsymbol{A}\right)^{2}\right]
$$

and is therefore diagonal with respect to the components of the vector potential. This implies that only average values of $\sin ^{2}(\kappa n z)$ and $\cos ^{2}(\kappa n z)$ will enter the formula for the Casimir energy and since these two averages are the same, the result reads:

$$
E_{\mathrm{QED}}^{(0)}=\sum_{n=1} \int \frac{d \omega}{2 \pi} \frac{d^{d} k_{\perp}}{(2 \pi)^{d}} \frac{\omega^{2}+\boldsymbol{k}_{n}^{2}}{2} \delta_{i j}\left(\delta^{i j}-\frac{\boldsymbol{k}_{n}^{i} \boldsymbol{k}_{n}^{j}}{\boldsymbol{k}_{n}^{2}}\right) \frac{i}{\omega^{2}-\boldsymbol{k}_{n}^{2}+i \delta} .
$$

Performing the sum over polarizations, we observe that $E_{\mathrm{QED}}^{(0)}$ is two times larger than the corresponding result for the scalar field in Eq.(7) and we obtain:

$$
E_{\mathrm{QED}}^{(0)}=-\frac{\pi^{2}}{720 L^{3}}
$$

Let us now turn to radiative corrections to this result. As we have seen from the scalar field theory example, the important thing is the expression for external potential. Since this 
potential depends on the boundary conditions and they are different for different components of the vector potential, the potential is sensitive to photon polarization.

To show the kind of expressions one gets, I consider the vacuum polarization diagram Fig.1a and the corresponding correction to the vacuum energy:

$$
\begin{gathered}
E_{\mathrm{QED}}^{(1 a)}=\sum_{n=1} \int \frac{d \omega}{2 \pi} \frac{d^{d} k_{\perp}}{(2 \pi)^{d}} \mathcal{F}(\kappa n, \Delta) \frac{\omega^{2}+\boldsymbol{k}_{n}^{2}}{2} \delta_{i j} D_{i i_{1}}\left(k_{n}\right) W_{i_{1} i_{2}}(\kappa n, \Delta) \Pi_{i_{2} j_{2}}\left(\omega, \boldsymbol{k}_{\perp}, \Delta\right) \\
\times W_{j_{2} j_{1}}(\kappa n, \Delta) D_{j_{1} j}\left(k_{n}\right),
\end{gathered}
$$

where $\Pi_{i_{2} j_{2}}$ is the vacuum polarization function, $D_{i j}(k)=i\left(\delta_{i j}-\boldsymbol{k}_{i} \boldsymbol{k}_{j} / \boldsymbol{k}^{2}\right) / k^{2}$ is the usual photon propagator in the Coulomb gauge, $\mathcal{F}$ is defined be the equation:

$$
\mathcal{F}=\frac{4}{L} \frac{(1-\cos (\kappa n L) \cos (\Delta L))}{\left[\Delta^{2}-(\kappa n)^{2}\right]^{2}},
$$

and the matrix $W$ reads:

$$
W_{i j}=\kappa n\left(\delta_{i j}-\boldsymbol{n}_{i} \boldsymbol{n}_{j}\right)+\Delta \boldsymbol{n}_{i} \boldsymbol{n}_{j}
$$

with $\boldsymbol{n}$ being the unity vector in the $z$ direction.

We see that the only significant change in the structure of the potential, as compared to the scalar case, is that now it changes the "polarization" of the photon in addition to the change in its momentum. Although this is an important modification, it does not affect our discussion of the relevant momentum regions in the previous Section. Therefore, the only momentum region that can give a non-zero contribution is the the momentum configuration where $\Delta$ is of the order of the electron mass while the photon momentum remains small. We can therefore proceed with the calculation of the effective Lagrangian in exactly the same way as in the previous Section, i.e. we consider the vacuum polarization diagram and integrate out "hard subgraphs" that include the lines of the external "potential". We then obtain the effective Lagrangian accurate to $\mathcal{O}(1 / m)$ :

$$
\delta \mathcal{L}_{Q E D}=\frac{3 \alpha}{32 L m}\left(-\boldsymbol{E}_{z}^{2}+\boldsymbol{B}_{\perp}^{2}\right) .
$$

Again, there are two sources of radiative corrections caused by this effective Lagrangian; the first one is the correction to the Green's function of the photon field and the second is the modification of the energy momentum tensor. The calculation of these corrections is now a simple matter and we easily reproduce the result of [5]:

$$
\delta E_{Q E D}^{(0)}=\frac{\alpha \pi^{2}}{2560 m L^{4}}
$$

The corresponding correction to the Casimir force is then:

$$
\frac{\delta f_{0}}{f_{0}}=-\frac{3}{8} \frac{\alpha}{L m}
$$




\section{CONCLUSION}

In this paper the radiative corrections to the Casimir force between two parallel plates are studied in both scalar field theory with one massive and one massless field and in QED. It is shown that, due to different quantization conditions for massive and massless fields, it is not possible to use effective field theories derived in the boundary-free case to get corrections to the Casimir force.

The picture that emerges from these considerations can be visualized by saying that the transition from massless to massive fields can not happen directly, but only through the interaction with the external potential. The expression for this potential is derived from the overlap integral of the eigenfunctions of heavy and light fields and is therefore boundary conditions dependent. Since the momentum transfer from the potential to quantum fields can be both large and small, one has to take this into account while constructing effective field theory for the calculation of the Casimir force by integrating out heavy degrees of freedom.

As can be expected from general considerations, the operators in the effective Lagrangian do not respect Lorentz invariance since the presence of plates breaks translation invariance in the $z$-direction. For the case of two interacting scalar fields, the effective Lagrangian is shown in Eq.(39). One sees explicitly that it is given by power series in $\left[\partial_{z}^{2} / M^{2}\right]$. The same can be seen from the effective Lagrangian for the QED Casimir effect given in Eq.(54).

Another remark concerns the dependence of the result on the boundary conditions. Consider the scalar field theory. In that case, all the results in the present paper correspond to Dirichlet boundary conditions for the massless field and one may wonder what will change if other boundary conditions are used. Let us choose von Neumann conditions $\left.\partial_{z} \phi\right|_{z=0}=\left.\partial_{z} \phi\right|_{z=L}=0$. In this situation the Green's function of the field $\phi$ changes. In spite of that, the leading order Casimir force remains the same. The radiative corrections to the Casimir force, however, do change; this happens because the function $\mathcal{F}_{D}(\kappa n, \Delta)$ depends on the boundary conditions. Such a dependence is not very surprising since large values of $\Delta$, that are responsible for the radiative corrections, correspond to the values $z \sim 0$ and $z \sim L$ and it is clear that the behavior of the field $\phi$ close to the plates is sensitive to the boundary conditions. In this respect the effective field theories for the Casimir force may be not that "effective" since the results of the present paper show that it is not possible to first integrate out heavy degrees of freedom and impose boundary conditions on remaining light degrees of freedom later on.

My final comment is on the relation of the results obtained in this paper and the calculation of radiative corrections to photon energy density at small temperature $T$. In fact, the calculation in Ref. [6] has been first performed for the finite temperature and later argued to be equivalent to the calculation of radiative corrections to the Casimir force once the substitution $T \rightarrow 1 /(2 L)$ is made. As I have shown above, the results of Ref. [6] related to radiative corrections to the Casimir force are based on the wrong effective field theory and for this reason are not valid. The natural question is then whether or not the finite temperature results of [6] are correct. To answer this question we have to recall that the formulation of the finite temperature field theories based on functional integral with certain boundary conditions on bosonic and fermionic degrees of freedom is just a (powerful) mathematical trick. However, this trick is not necessary. To compute the energy density 
of the photon field, one can imagine constructing (without any restrictions on space-time) an effective Hamiltonian first and taking thermal average over the photon states later. It is crucial that no reference to boundary conditions appears in the process of constructing effective Hamiltonian in this case and therefore heavy degrees of freedom can be integrated out in the boundary-free manner. It is also physically sound to take thermal averages over the photon states or, equivalently, impose periodic boundary conditions on the photon field in the functional integral after heavy particles have been integrated out 0 . This is exactly the procedure that has been followed in [6] and the above comments show that it is meaningful in case of the finite temperature. It is, however, not at all meaningful for the Casimir effect where first boundary conditions should be imposed and only after that should the heavy particles be integrated out. In my opinion, the main result of this paper is a clear demonstration of the fact that these two procedures do not commute.

\section{ACKNOWLEDGMENTS}

This work was supported in part by DOE under grant number DE-AC03-76SF00515. I am grateful to Lance Dixon and Marvin Weinstein for many useful conversations.

\section{APPENDIX}

Some formulas used in the derivation.

$$
\begin{gathered}
I_{0}(a, b)=\int \frac{d^{d} p}{(2 \pi)^{d}}\left(\frac{1}{p^{2}+1}\right)^{a}\left(\frac{1}{p^{2}}\right)^{b}=(4 \pi)^{-d / 2} \frac{\Gamma(a+b-d / 2) \Gamma(d / 2-b)}{\Gamma(a) \Gamma(d / 2)}, \\
\zeta(-2 n-3)=\frac{(-1)^{n} \zeta(2 n+4)(2 n+3) !}{\pi^{2 n+4} 2^{2 n+3}}, \\
\Gamma\left(z+\frac{1}{2}\right)=\frac{\sqrt{\pi}}{2^{2 z-1}} \frac{\Gamma(2 z)}{\Gamma(z)}, \quad \int_{0}^{1} \frac{\mathrm{d} x \ln ^{n} x}{1-x}=(-1)^{n} n ! \zeta(n+1) .
\end{gathered}
$$

\footnotetext{
${ }^{5}$ Note that in this case the photons and the electrons are not in thermal equilibrium.
} 


\section{REFERENCES}

[1] H. B. G. Casimir, Proc. K. Ned. Akad. Wet. 51, 793 (1948).

[2] S. K. Lamoreaux, Phys. Rev. Lett. 78, 5 (1997).

[3] U. Mohideen and A. Roy, Phys. Rev. Lett. 81, 4549 (1998).

[4] For a review, see K. Milton, hep-th/9901011.

[5] M. Bordag, D. Robaschik and E. Wieczorek, Ann. Phys. 165, 192 (1985); D. Robaschik, K. Scharnhorst and E. Wieczorek, Ann. Phys. 174, 401 (1987); M. Bordag and K. Scharnhorst, Phys. Rev. Lett. 81, 3815 (1998).

[6] X. Kong and F. Ravndall, Phys. Rev. Lett. 79, 545 (1997); Nucl.Phys. B526, 627 (1998).

[7] F. Ravndall, hep-ph/0009208.

[8] C. A. Lütken and F. Ravndal, Phys. Rev. A31, 2082 (1985). 


\section{FIGURES}

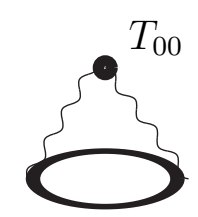

(a)

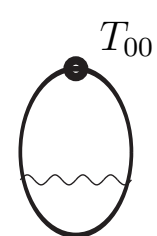

(b)

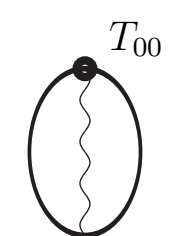

(c)

FIG. 1. One loop corrections to the vacuum energy. The wavy and bold solid lines represent the massless and the massive particles, respectively. Insertions of the stress-energy tensor are shown explicitly.

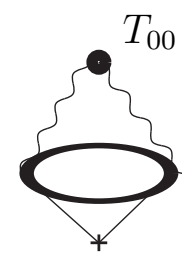

FIG. 2. One loop correction to the vacuum energy with "external potential" lines shown explicitly. Momentum configuration that produces non-trivial Wilson coefficients corresponds to the large $\sim M$ momenta flowing through the massive and external potential lines. 